\title{
Convective Overshooting to Drive g-Modes in $\gamma$ Dor Stars
}

\author{
Y. Li \\ Yunnan Observatory, NAOCAS, P.O. Box 110, Kunming, 650011 China
}

\begin{abstract}
From considerations of kinetic energy input from turbulent convection into g-mode pulsation, we show that g-mode pulsation may obtain enough energy from the turbulence to overcome damping in the stellar interior. The development of thick convective envelope in the stellar models requires a small value of the convection parameter $\alpha=1.2$ to ensure the low mass limit of the $\gamma$ Dor stars' instability strip to be in agreement with the observations.
\end{abstract}

\section{Physical Assumptions and Evolution Models}

Great interests arise recently from the discovery of g-mode oscillations in the $\gamma$ Doradus stars, which provide an unique chance to probe the cores of these stars(Krisciunas 1998; Kaye et al. 1999; Handler 1999). A major difficulty to explain these pulsating stars is how they are driven to pulsate? The well-known $\kappa$-mechanism is shown not be able to be responsible for the observed pulsation (Gautschy \& Löffler 1996). In the present work we investigate the interaction between convection and g-mode pulsation as a possible driving mechanism.

First of all, it should be noticed that stellar convection and g-mode pulsation are driven by the same force, the buoyancy. When the buoyancy is on the opposite direction of the motion, it pulls back the fluid elements to form g-mode pulsation; on the other hand, the convection develops. From this basic picture we may expect the production of g-mode pulsation, when convection cells come out of the convection zone known as convective overshooting phenomenon. On the other hand, when a pulsating element goes into the convection zone, it becomes then a convection cell and will continously move forward and mix with the environment after a mixing length. So the pulsation is dissipated through this nonlinear interaction. However if the convection zone is not too much thick, namely thinner than a mixing length, many convection cells may servive from annihilation in the convection zone and escape to form effective convective overshooting. Such a thin convection zone may serve as driving domain of the g-mode pulsation.

To verify the effectiveness of the above mechanism for the $\gamma$ Dor phenomenon, we calculate stellar models using updated input physics. The evolution tracks of these models are plotted on the HR diagram in Figure 1. It can be found that $1.5-1.8 M_{\odot}$ models cover the location of the observed variable stars. Different values of the convection parameter $(\alpha=1.8$ or 1.2$)$ result in trivial influence on the evolution tracks that we are interested. Figure 2 shows the distribution of the Brunt-Väisälä frequency $N$ in these models. The g-mode 


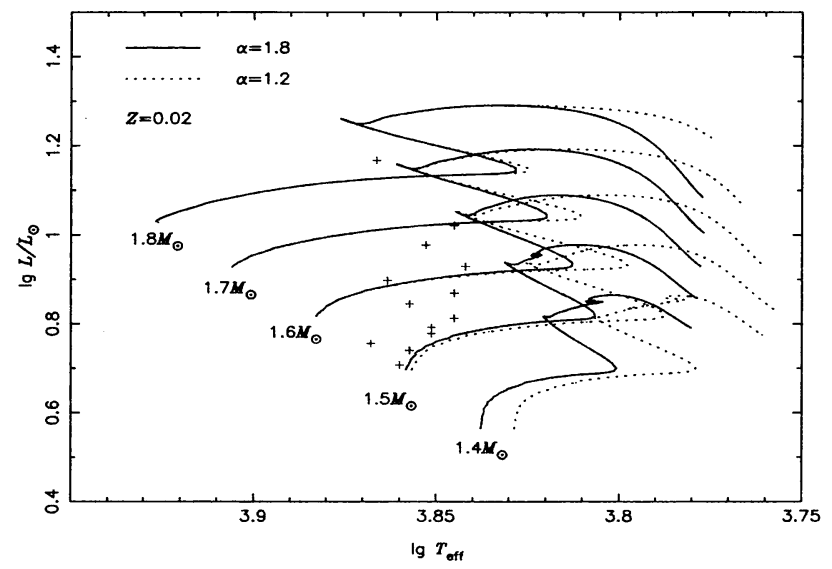

Figure 1. Evolution tracks of the $\gamma$ Dor stars. Some observed $\gamma$ Dor stars from Kay et al. 1999 are also plotted

pulsation can propogate in regions where $N$ is positive. In the convection regions $N^{2}$ is negative and we plot a negative value to indicate this case. We notice that some considered stellar models indeed have two convection zones in their envelopes. Each convection shell is only of the thickness $0.5-0.8$ times the local pressure scale height, which is considerably shorter than the mixing length. Based on the arguments discussed above, g-mode pulsation may experience driving effect and be able to get out of the convection shells before seriously dissipated by convective annihilation. However, models with convection parameter $\alpha=1.8$ and mass smaller than $1.6 M_{\odot}$ develop thick convection envelopes, and are not suitable for g-modes, if generated below the base of the convection zone, to penetrate onto the stellar surface. Integrating this fact with the evolution tracks in Figure 1, we may conclude that models with convection parameter $\alpha=1.8$ cannot explain the lower effective temperature boundary of the observed variable stars on the HR diagram. When the convection parameter is chosen to be $\alpha=1.2$, we find that stellar models with mass smaller than $1.5 M_{\odot}$ develop thick convection envelopes, and this result defines lower effective temperature boundary of the pulsation instability strip that is in good agreement with the observations.

\section{Pumping Effect of Thin Convection Shell}

As discussed in the above section, thin convection shell may amplify the gmode pulsation penetrating through it. If the convective driving is comparable to the damping of the pulsation, the pulsation may have enough amplitude to be observable. we call this mechanism as the convective pumping effect. The strength of this mechanism can be estimated as follows. The total kinetic energy a g-mode pulsation can obtain from the turbulence is estimated to be

$$
K_{t k}=4 \pi r^{2} \rho v^{3} \frac{l}{v}
$$


where $\rho$ is the density, $v$ the typical velocity of the turbulence, and $l$ the mixing length of the convection. The average accelerating time is estimated to be $l / v$. On the other hand, the total kinetic energy of a pulsation mode is

$$
E_{k}=\int \frac{1}{2} \rho \sigma^{2}|\xi|^{2} d V
$$

where $\sigma$ is the frequency and $\xi$ the displacement of the pulsation. Table 1 lists these quantities for some computed models. Comparing the values of $K_{t k}$ and $E_{k}$ that represent respectively the energy that the convection zone can supply for the pulsation and the inertia of the pulsation mode, we find that one time of convective pumping does not supply a mode with enough kinetic energy to achieve an observable amplitude. But the pumping time is a few tenth of the pulsation period, and the period is a few hundredth of the life time. This means that continous operating of the convective pumping is able to supply enough kinetic energy for a mode to be observable. We notice also that a shorter period mode usually has a longer lifetime and a smaller mode inertia, which implies it easier to be excited.

Table 1. Comparison of pulsation excitation for $\gamma$ Dor stars

\begin{tabular}{lllllll}
\hline$M / M_{\odot}$ & $R / R_{\odot}$ & $T_{\text {eff }}(\mathrm{K})$ & $P($ day $)$ & $\tau($ day $)$ & $E_{k}(\mathrm{ergs})^{\#}$ & $K_{t k}(\mathrm{ergs})$ \\
\hline \hline 1.5 & 1.52 & 7143 & 1.04 & 51.8 & $1.59 \times 10^{36}$ & $7.01 \times 10^{35}$ \\
& & & 2.05 & 41.9 & $6.27 \times 10^{41}$ & \\
& & & 2.94 & 23.8 & $2.30 \times 10^{52}$ & \\
\hline \hline 1.8 & 2.58 & 6912 & 1.19 & 1680. & $1.07 \times 10^{38}$ & $4.32 \times 10^{36}$ \\
& & & 2.38 & 83.5 & $3.26 \times 10^{37}$ & \\
& & & 3.25 & 92.4 & $8.81 \times 10^{40}$ & \\
\hline
\end{tabular}

\# Assuming $0^{\mathrm{m}} .001$ amplitude

\section{Conclusions and Discussions}

Convection is a critical factor in explaining the pulsation observed in the $\gamma$ Dor stars . Large scale convection zones act as efficient damping domains of the g-mode oscillations, while thin convection shells may amplify the g-mode pulsation penetrating through them. From considerations of kinetic energy input from turbulent convection into g-mode pulsation, we show that the convective pumping mechanism may supply enough energy for the g-mode pulsation to overcome damping in the stellar interior. As the convection shell is dynamical unstable region, any g-mode incident into it will experience driving effect, and the convective pumping mechanism prefers multimode excitation. On the observations, being multimode pulsator is a chief character that distinguishes the $\gamma$ Dor stars as a new type of pulsating variables. In the meantime, the convective motion is always unstable, leading to variable amplitude of the excited g-modes that is another observed property.

Another result is that the development of thick convective envelope in the stellar models requires a small value of the convection parameter $\alpha=1.2$ to 

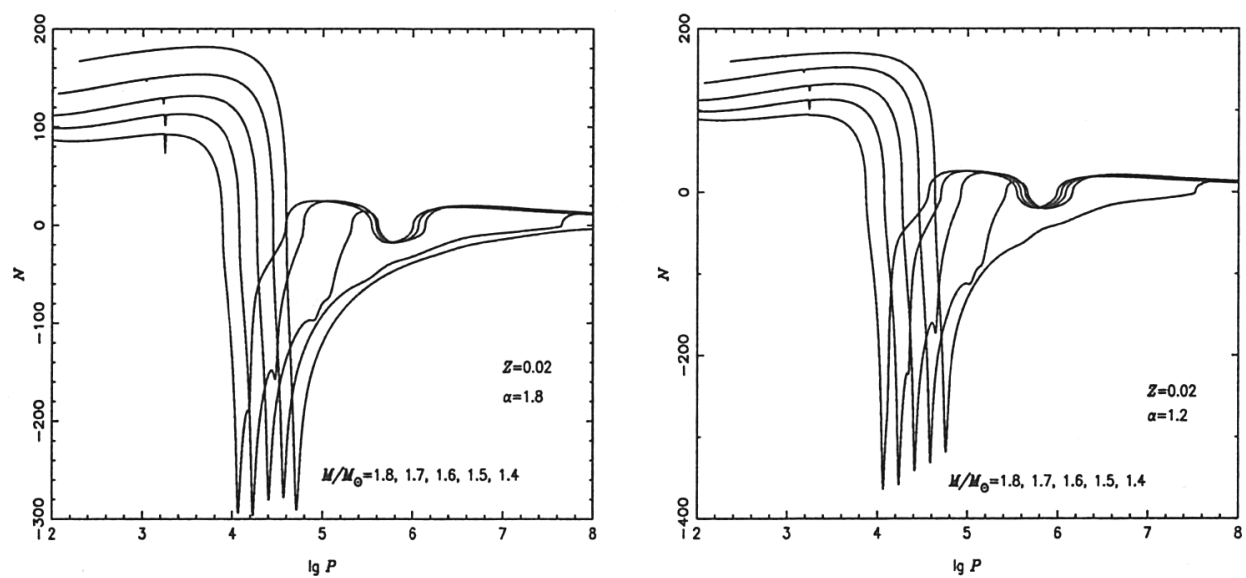

Figure 2. Distribution of the Brunt-Väisälä frequency $N$ in the $\gamma$ Dor models. $N$ is plotted against $\lg P$ in unit of cycle per day

ensure the low mass limit of the $\gamma$ Dor stars' instability strip down to the observed place of about $1.5 M_{\odot}$. This forms a sharp contract to the case of the solar models in which $\alpha$ is found to be around 1.7 (see, for example, Guzik \& Swenson 1997). The reason may be understood as follows. Compared with the fully developed convection in solar envelope, the convection in the $\gamma$ Dor stars is very underdeveloped. The thickness of their convection shells is smaller than the local pressure scale height. We may expect that mixing in the convection shells of the $\gamma$ Dor stars is not sufficient, which decreases the efficiency of energy transport by convection and leads to a small value of the convection parameter $\alpha$. This result can then be regarded as a rough determination of the convection parameter $\alpha$ in the $\gamma$ Dor stars by the seismic methodology.

Though we suggest in this paper the possibility of convective overshooting as the driving mechanism of the $\gamma$ Dor phenomenon, more work should be done to clarify the details of its operation. The author is in great debt to H.Y. $\mathrm{Xu}$ for fruitful discussions and collection of some relevant literature and to J.Y. Yang for preparation of the evolution code. This work is sponsored by the NSFC through project number 19625306 and 19833040, and National Key Fundamental Research Project "Formation and Evolution of Galaxies".

\section{References}

Gautschy, A., \& Löffler, W., 1996, Delta Scuti Stars Newsletter, 10, 13

Guzik, J.A., \& Swenson, F.J., 1997, ApJ, 491, 967

Handler, G., 1999, MNRAS, 309, L19

Kaye, A.B., Handler, G., Krisciunas, K., Poretti, E., \& Zerbi, F.M., 1999, PASP, 111,840

Krisciunas, K., 1998, in New Eyes to See Inside the Sun and Stars, IAU Symp. 185, eds. F.-L. Deubner et al., p.339 